\title{
Aspectos tectónicos con relación a la geología local en el sector de k'uypan en Ocopata un estudio de caso
}

\section{Tectonic aspects in relation to local geology in the $k^{\prime} u y p a n$ sector in Ocopata a case study}

\author{
Yessenia Puma ${ }^{1}$, Yenifer Mamani ${ }^{1}$, Diana Lozano ${ }^{1}$ \\ ${ }^{1}$ Universidad Nacional de San Antonio Abad del Cusco, Cusco, Perú, \\ Correoelectrónico:170401@unsaac.edu.pe
}

\begin{abstract}
Resumen
El presente trabajo de investigación tuvo como objetivo principal determinar la geología local y su influencia tectónica del sector K'uypan Occopata Cusco, que presentó secuencias sedimentarias de las formaciones Puquin, Chilca, Quilque y la formación Kayra, de los cuales se determinó a una escala 1/5500 los contactos inferidos, debido al alto tectonismo dentro del área de estudio y al cambio brusco de litología. Para comprender la parte estructural del sector $\mathrm{K}$ 'uypan se realizó el estudio de imágenes satelitales esto para entender la dinámica y el control estructural regional que es de mucha importancia para la interpretación de los diferentes eventos geológicos y tectónicos, luego en campo se corroboró con el mapeo geológico- estructural local, donde se pudo observar los controles estructurales en dirección NW-SE con mayor predominancia en el lugar, así también fallas de tipo normales, inversas, sinextrales y dextrales.
\end{abstract}

Palabras Clave: Tectónica; geología; estructuras geológicas; contactos litológicos; discordancia.

\begin{abstract}
The objective of this research work was to determine the local geology and its tectonic influence of the K'uypan Occopata Cusco sector that presented sedimentary sequences of the Puquin, Chilca, Quilque and Kayra Formations, of which it was determined at a scale of $1 / 5500$ the inferred contacts, due to the high tectonism within the study area and the abrupt change in lithology. To understand the structural part of the K'uypan sector, the study of satellite images was carried out to understand the dynamics and regional structural control that is of great importance for the interpretation of the different geological and tectonic events, then in the field it was corroborated with the local geological-structural mapping, where it was possible to observe the structural controls in the NW-SE direction with greater predominance in the place, as well as normal, inverse, synextral and dextral faults.
\end{abstract}


Key words: Tectonics; geology; geological structures; lithological contacts; discordance.

\section{Introducción}

La geología y tectónica del área de estudio ubicada dentro del Altiplano (INGEMMET, 2011), al suroeste de la ciudad de Cusco, distrito Santiago, comunidad Occopata, sector K'uypan y que se encuentra a unos 25 minutos de la ciudad de Cusco (Ver Mapa $\mathrm{N}^{\circ} 1$ ), vienen ligados a procesos de geodinámica interna (fallas, anticlinales, sinclinales) y geodinámica externa (erosión, deslizamientos antiguos y recientes) (GAMBOA, 2015).

El presente trabajo se realiza para determinar las diferentes características tectónicas y geológicas de la zona y de ese modo realizar una interpretación tectónica y geológica mediante el análisis de datos estructurales en el sector, siendo de importancia verificar los diferentes procesos que existieron en el sector; además se menciona que el sector de K'uypan-Occopata posee fallamientos y plegamientos inusuales (distintas direcciones de fallas normales e inversas, plegamientos volcados).

\section{$\operatorname{Mapa} \mathbf{N}^{\circ} 1$}

Mapa de ubicación del Sector de K'uypan (elaborado en el programa ArcGIS)

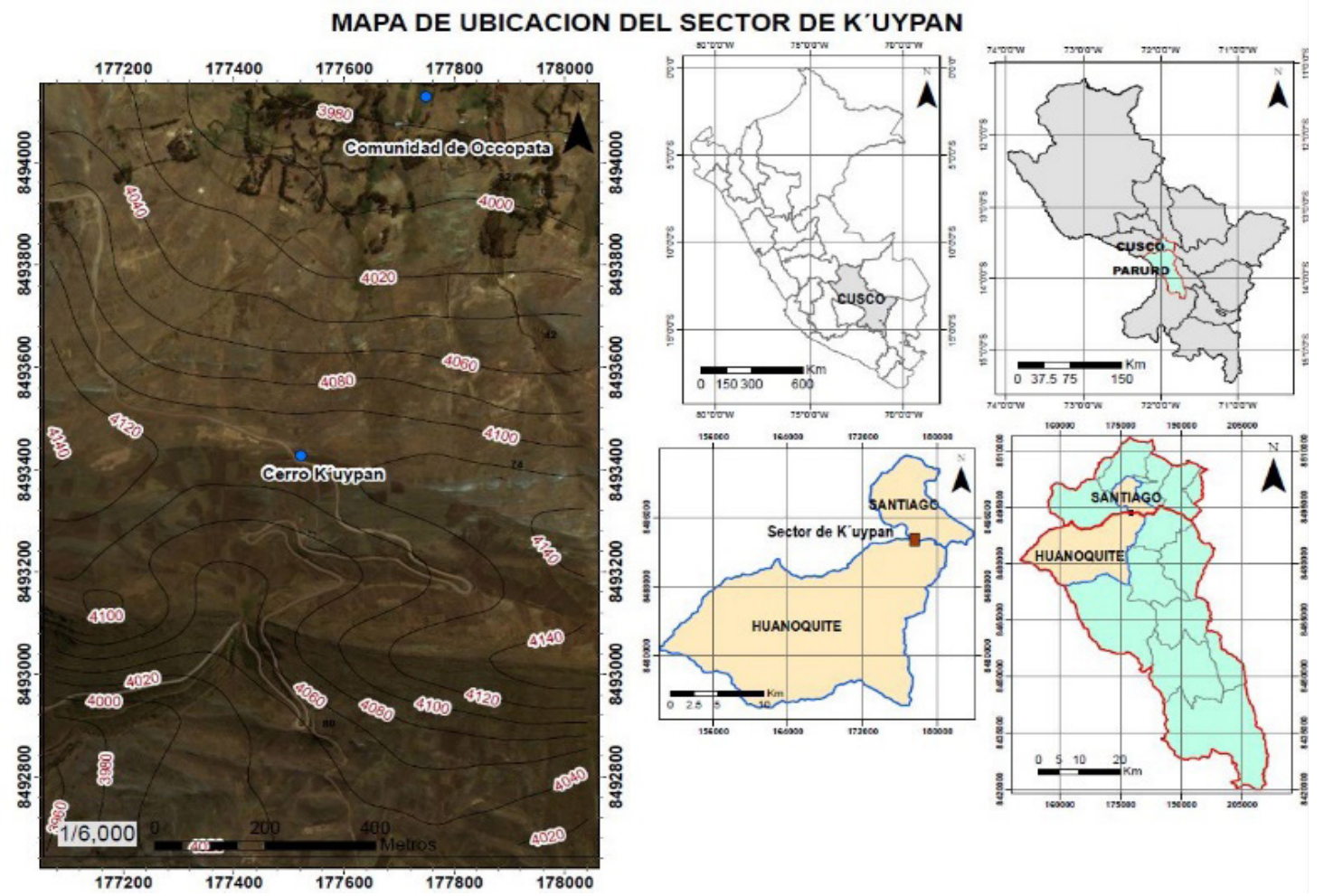




\section{Metodología}

El área de estudio de la zona está en base al cartografiado geológico-estructural local representado por las formaciones Puquin, Quilque, Chilca, y Kayra, con diferentes edades de atribución, que no cumple la disposición del más antiguo al más reciente debido a la presencia de tectonismo caótico dentro de la zona.

\section{Aspectos geológicos}

En el sector K'uypan la sedimentación está compuesta por depósitos con variadas litologías. Se identificó la formación Puquin en sus Miembros M2 y M3 pertenecientes a la edad Coniciano-Maestrichtiano (Carlotto, 1992; Carlotto et. Al., 1992) así mismo; se identificó la Fm. Quilque del Paleoceno inferior (Gregory. 1996; Carlotto, 1992), la Fm. Chilca de edad del Paleoceno superior al Eoceno basal (Audebaud, 1973; Carlotto, 1992) y la Fm. Kayra del grupo San Jerónimo de edad Eoceno inferior (Córdova, 1986; Carlotto, 1998)

\section{Formación Puquin.}

La Fm. Puquin sobreyace a la formación Ayabacas, está dividida en tres miembros (miembro 1, 2, 3) y aflora ampliamente en el núcleo del anticlinal de Puquin, en el anticlinal de Saylla, al norte de Saylla, entre Rondocan y San juan Quihuares al norte de Accha. (Carlotto, 2011)

En la zona de estudio, dicha formación aflora mayormente el miembro M2, ya que se encontró una secuencia basal conformada por lutitas negras ricas en materia orgánica, turgita y presencia de zonas de oxidación, con intercalaciones de arenisca feldespática de grano fino de color gris claro, mientras que la parte media y superior está conformada por lutitas arenáceas de color verde, areniscas cuarzosas de grano medio con presencia de costras de calcita y talco. (Ver Figura $\mathrm{N}^{\circ} 1$ )

El miembro M2 aflora ampliamente en el anticlinal de Puquin y sobreyace al miembro M3. Sin embargo, este último se halla parcialmente erosionado (Carlotto, 2011). Es por ello que en la zona de estudio encontramos el M2.

\section{Formación Quilque.}

Según (Carlotto, 2011), la formación Quilque, aflora al oeste de la ciudad de Cusco en los flancos del anticlinal de Puquin, y en los flancos de los anticlinales de Saylla, Occopata y Sondor. Dicha formación es un conjunto de más de $150 \mathrm{~m}$ de espesor, con una disposición grano estratocreciente de lutitas, areniscas de color rojo y conglomerados en la parte superior, estos últimos formados por la erosión de costras calcáreas y calizas. Al suroeste de la zona de estudio en el sector K'uypan- Occopata (UTM: 177893E-8493384N) esta formación aflora en una secuencia grano estratodecreciente y se encontró en la parte basal microconglomerados seguido de areniscas calcáreas, margas y lutitas de tonalidades rojas y verdes (Ver la Figura $\mathrm{N}^{\circ} 1$ )

Los estratos están en una disposición grano estratodecreciente, siendo la base arenisca feldespática de grano medio a grueso.

\section{Formación Chilca.}

Según (Carlotto, 2011) esta formación de más de 100m de espesor, se encuentra en discordancia erosional sobre la formación Quilque y bajo la formación Kayra. Al sureste de la zona de estudio en el sector K'uypan- Occopata UTM: (177953E-8493637N) dicha 
formación aflora con una secuencia de lutitas de tonalidades rojas con yeso diseminado, areniscas carbonatadas de color gris blanquecinas que pasan gradualmente a areniscas feldespáticas de color rojo (Ver la Figura $\mathrm{N}^{\circ} 1$ )

\section{Formación K'ayra.}

La formación K'ayra pertenece a las capas rojas del grupo San Jerónimo, aflorando ampliamente al suroeste de Cusco donde forma parte del sinclinal de Anahuarqui y anticlinal de Puquin al oeste de Cusco (Córdova, 1986).

En la zona de estudio aflora la formación K'ayra al sureste del sector Occopata, llegando a los linderos de la comunidad Checcapuca, dicha formación se encuentra en contacto erosional inferido con la formación Chilca UTM (177473E-8493026N). La secuencia estratigráfica que sigue es grano creciente, en la base se encuentra una secuencia de lutitas rojas y areniscas feldespáticas de grano fino a medio, y la parte media-superior conforma areniscas de grano grueso con clastos blandos de lutita y conglomerados.

\section{Figura $\mathbf{N}^{\circ} 1$}

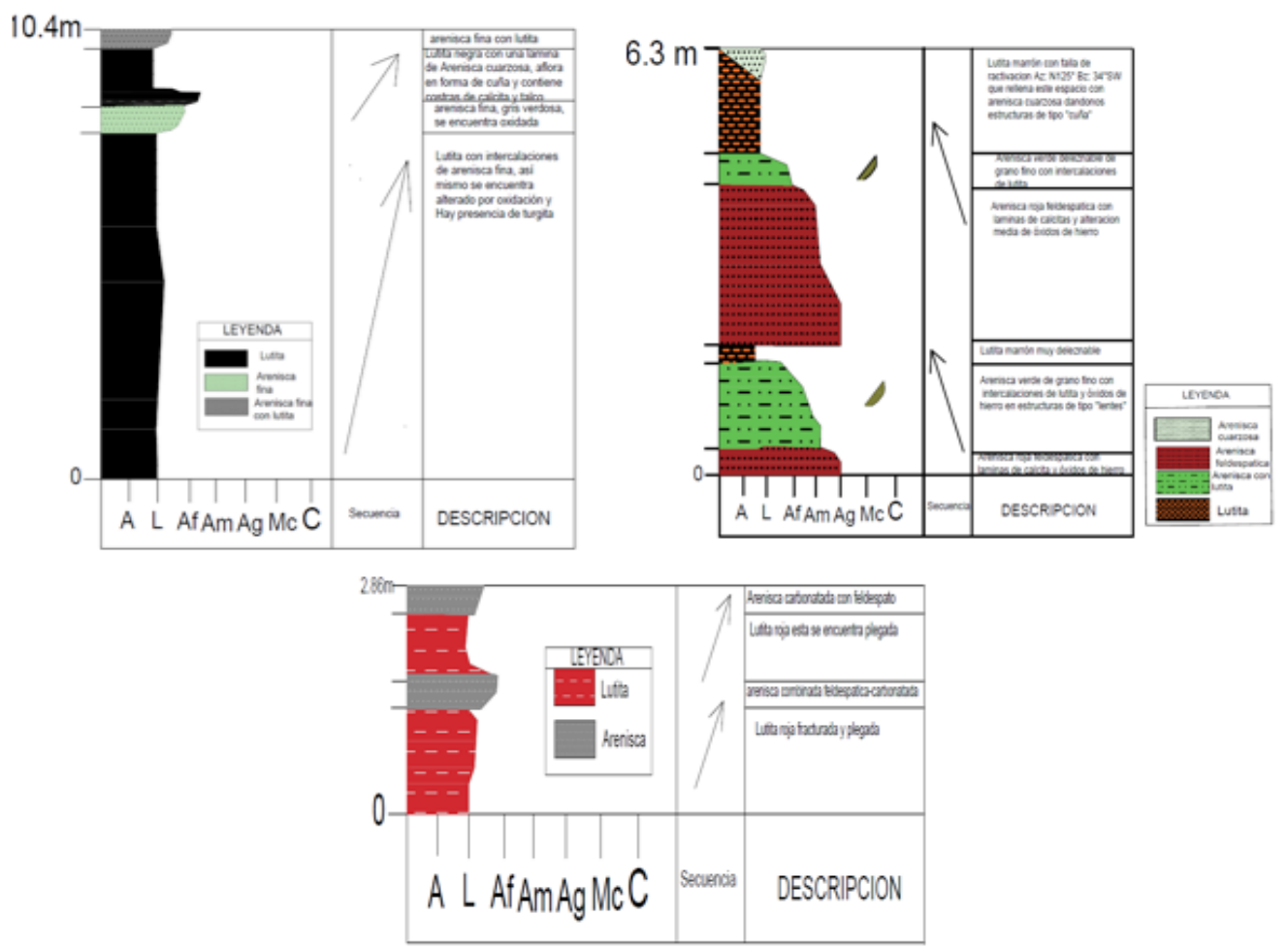

Nota. Columnas estratigráficas, formación Puquin, miembro 2, tomada al SE del sector Occopata

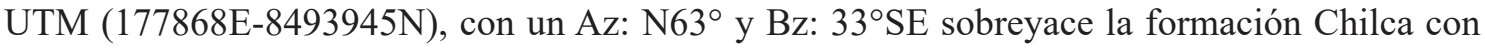
un Az: $\mathrm{N} 290^{\circ}$ y Bz: $42^{\circ} \mathrm{NE}$ (parte superior izquierda), formación Quilque tomada en el corte de carretera al SW del sector Occopata UTM (177603E-8493290N) (parte superior derecha) y formación Chilca al SE del sector Occopata UTM (177953E-8493637N) con una disposición grano estratocreciente, lo característicos es que las areniscas son feldespáticas carbonatas a diferencia de la Fm, Quilque (parte inferior central). ((Elaborado en el programa AutoCAD 2020). 


\section{Aspectos Tectónicos.}

El área de estudio se encuentra en una zona de mucha deformación, por el cual se presencia muchas estructuras geológicas. Así el área de estudio se encuentra limitado estructuralmente al norte por la presencia de la falla activa Ayarate-San Juan de Quihuares que hace cabalgar a las diferentes formaciones en dirección Sur, así también al Sur se encuentra limitado por la falla Paruro, el cual realiza un movimiento de cizalla y cambia la dirección de las formaciones aproximadamente $90^{\circ}$, por su parte al noroeste del área de estudio se pudo ver la presencia de formaciones que pertenecen al núcleo del anticlinal de Puquin (formación Puquin M2) que estaría interviniendo en este proceso de cambio de dirección de la zona de estudio, esto debido al movimiento de compresión del flanco que tiene dirección SW de la ciudad de Cusco.

\section{Control Estructural.}

El control estructural de la zona de estudio está en dirección NW-SE mayormente (Ver Figura $\mathrm{N}^{\circ}$ 2). Aunque encontramos estratos que afloran de forma inversa debido al movimiento caótico y cambio de dirección E-W de las formaciones por la dinámica de los esfuerzos compresivos de la zona estudiada; como estructuras de orden menor dentro del área de la formación Quilque tenemos fallas normales lístricas de tipo "domino" que toman menor importancia de acuerdo al movimiento de las estructuras en toda la zona.

\section{Figura $\mathrm{N}^{\circ} 2$}

\begin{tabular}{|l|l|l|}
\hline ID & Dip & Dip Direction \\
\hline 1 & 345 & 10 \\
\hline 2 & 125 & 34 \\
\hline 3 & 125 & 34 \\
\hline 4 & 217 & 90 \\
\hline 5 & 285 & 57 \\
\hline 6 & 345 & 10 \\
\hline 7 & 45 & 45 \\
\hline 8 & 97 & 62 \\
\hline 9 & 195 & 25 \\
\hline 10 & 260 & 20 \\
\hline 11 & 335 & 90 \\
\hline 12 & 280 & 57 \\
\hline 13 & 80 & 42 \\
\hline 14 & 53 & 65 \\
\hline
\end{tabular}

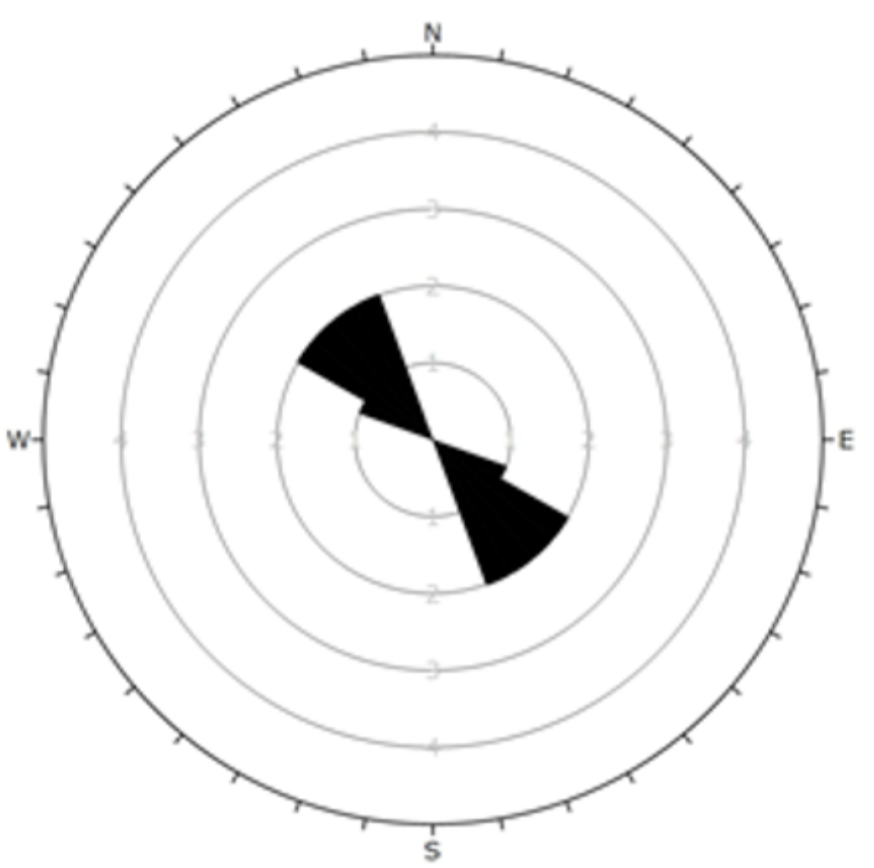

Nota. Estructuras compresionales de orientación NW-SE debido a la acción de las estructuras mayores que limitan en las diferentes direcciones a la zona de estudio, produciendo fallas inversas al norte, fallas normales al sur y un movimiento de rumbo de EW (Elaborado en el programa Dips 6.0) 


\section{Instrumentos}

La presente investigación tiene como instrumentos de campo una brújula de mano, picota, lupa, flexómetro, escalímetro, bolsas de muestreo, GPS, mapa satelital (Google Earth Pro), rayador y para el procesamiento de datos se usó el programa Excel, AutoCAD, ArcGIS 10.5 y Dip 6.0)

\section{Análisis de datos}

Primeramente, los datos fueron insertados en el programa Excel, luego con estos datos se elaboró las columnas estratigráficas en el programa AutoCAD 2020, posteriormente algunos datos del Excel fueron procesados en el programa de ArcGuis 10.5 que nos permiten la elaboración de mapa base, mapa geológico-estructural y la sección geológica, finalmente con datos estructurales incluidos en el Excel se procesaron en el programa Dip 6.0.

\section{Resultados}

La tectónica juega un papel importante para que se dé una inversión estratigráfica en el sector de $\mathrm{K}^{\prime}$ uypan, razón por el cual se encuentran aflorando formaciones más antiguas a las más recientes.

Basándonos en el cuadrángulo del cusco 28-s del INGEMMET, donde indica que, al noroeste de la ciudad del cusco, se ubica el anticlinal de Puquin. Posteriormente a este evento se dio una nueva activación de esfuerzos regionales, y dio como consecuencia el desarrollo de diferentes fallas, estos esfuerzos vienen del Norte y del Oeste, generando un desvío del anticlinal Puquin dando un giro aproximadamente 90 grados y comprime este anticlinal produciendo cabalgamientos en las formaciones. Razón por el cual hemos encontrado y medido fallas inversas que tienen un comportamiento tanto dextral como sinextral. Después se dio un periodo de relajamiento, por tal motivo se generan las fallas normales con una orientación $\mathrm{N} 86^{\circ}$ y buzamientos en dirección $\mathrm{SE}$, así mismo se encontraron formaciones que buzan al norte con un azimut aproximado de $\mathrm{N} 280^{\circ} \mathrm{y}$ siguiendo la dirección NS se encontró la siguiente secuencia: Fm. Puquin, Fm Chilca, Fm Quilque, Fm Chilca finalmente la Fm. Kayra como se observa en sección № 1. 


\section{$\operatorname{Mapa} \mathbf{N}^{\circ} 2$}

Mapa geológico estructural local del sector de K'uypan a una escala 1/5500 (elaborado en el programa ArcGIS 10.5)

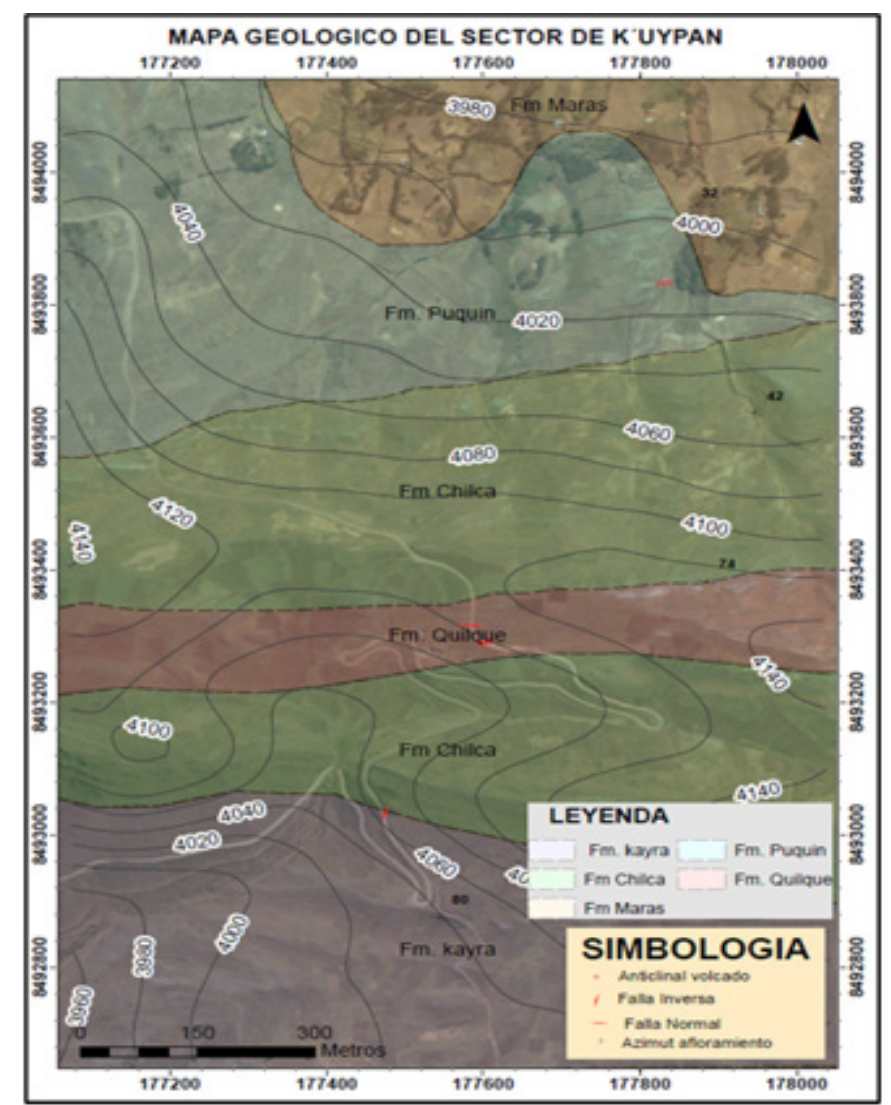

\section{Sección $\mathbf{N}^{\circ} 1$}

Perfil geológico del sector de K'uypan (elaborado en el programa ArcGIS 10.5).

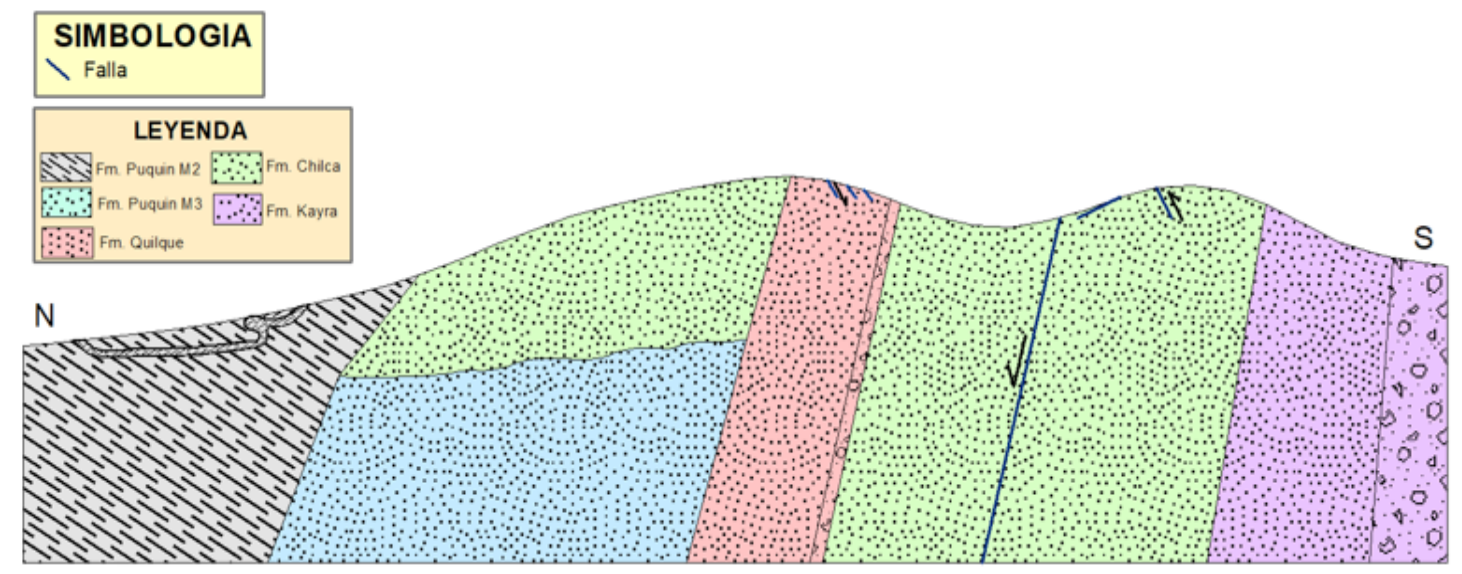




\section{Conclusiones}

Se definieron contactos litológicos inferidos a una escala 1/5500 entre las formaciones Puquin M2-Chilca, Chilca-Quilque y Chilca-Kayra, que debido a la tectónica ocurrida, la formación Quilque se encuentra invertida con una disposición grano estratodecreciente.

Se determinó estructuras compresionales de orientación NW-SE debido a la dinámica de las estructuras mayores que limitan en las diferentes direcciones a la zona de estudio, produciendo fallas inversas al norte, fallas normales al sur y un movimiento de rumbo de EW debido al cabalgamiento de la Ayarate-San Juan de Quihuares.

Se interpretó la relación que existe entre la tectónica y la geología del sector de K'uypan, por acción de los esfuerzos tectónicos, provenientes de norte y del oeste. Girando y comprimiendo el anticlinal de Puquin generando una inversión estratigráfica con cabalgamiento. Posteriormente en un periodo de relajación se produce cambios de buzamientos de las formaciones. Teniendo así la siguiente secuencia: Fm. Puquin, Fm Chilca, Fm Quilque, Fm Chilca finalmente Fm. Kayra.

\section{Recomendaciones}

- Realizar caminatas y exploraciones por toda el área de estudio, ya que no solo encontraremos los contactos de las formaciones en corte de carreteras sino también en puntos dentro del área de estudio donde no hay acceso vehicular.

- Desarrollar estudios en otros ámbitos de la geología, uno de ellos la geología económica, ya que se encontró presencia de fracturas mineralizadas con un posible potencial económico.

- Realizar un estudio más amplio incluyendo todos los sectores que han sido afectados por la acción de esfuerzos compresionales mayores que limitan el área de estudio.

\section{Declaración de conflicto de intereses}

Los autores declaran no presentar ningún tipo de conflicto de intereses. 


\section{Referencias}

Aguirre, E., Benavente, C., Zerathe, S., Audin, L., Delgado, G., \& Garcia, B. (s.f.). Análisis morfo-estructural cuantitativo en la falla activa Purgatorio-Mirave: antearco de los andes centrales - sur de Perú. Lima: Instituto Geológico Minero y Metalúrgico.

Baldellon, E., Fornari, M., Espinoza, F., \& Soler, P. (1994). SUCESION ESTRUCTURAL DE LA ZONA SERRANIA DE LAS MINAS. La Paz: instituto de investigacion Geológico.

Benavente Escóbar, C., Delgado Madera, F., Taipe Maquerhua , E., Audin , L., \& Pari Pinto, W. (2013). Neotectónica y Peligro Sísmico en la Región Cusco. Lima.

Boulanggerr Rondoy, E., Chong Kam, L. M., \& Sipión Baltodano, C. (2019). Estudio de recursos de rocas y minerales industriales para la inclusión económica social y desarrollo en la región Cusco: sector meridional. 2019: INGEMMET, Boletín Serie B: Geología Económica.

CANLLAHUI DURAN, J. C. (2018). ANÁLISIS ESTRUCTURAL Y SU RELACIÓN CONEL EMPLAZAMIENTO DE LA MINERALIZACIÓN ENEL PROYECTO CRUCERO - PUNO. Puno.

Carlotto Caillaux, V., Cárdenas Roque, J., \& Carlier, G. (2011). Geologia del cuadrángulo de Cusco Hoja 28-S. Lima Perú.

Vilchez, M., \& Lacroix, P. (2015). ÚLTIMA REACTIVACIÓN DEL MOVIMIENTO COMPLEJO DE RANRACCASA-YAURISQUE-PARURO-CUSCO. Instituto Geológico, Minero y Metalúrgico - INGEMMET, 110-113. 